\title{
Connectivity and cognition in neuropsychiatric disorders with special emphasis on Alzheimer's disease and Chorea Huntington
}

\author{
Dorota Badowska • Andrea Schmitt • \\ Peter Falkai
}

Published online: 1 July 2014

(c) Springer-Verlag Berlin Heidelberg 2014

This issue opens with an invited review by Teipel et al. [1] discussing the use of diffusion tensor imaging (DTI) in the highly comorbid Alzheimer's disease (AD) and affective disorders. DTI allows the examination of structural brain connectivity and until now has been widely used to investigate impairment of intracortical projection tracts, which has been hypothesized to be the basis of cognitive dysfunction. Analyses of large scale data revealed white matter abnormalities in AD. Fewer DTI studies focused on affective disorders, e.g., bipolar disorder and major depressive disorder with inconsistent results. Larger and more homogenous groups of patients would allow the detection of subtle differences and avoid false-negative results. Applying other techniques could also open new possibilities for studying the brain network. Diffusion spectrum imaging (DSI) offers better resolution than DTI, but is rarely used due to its limited availability, software requirements and long duration of analysis. More common use of DSI would improve data quality and provide better insight into the structural connectivity in neuropsychiatric diseases. Additionally, prospective studies are needed to follow-up patients with $\mathrm{AD}$ and mild cognitive impairment for several years. Understanding the changes that prelude the symptoms would allow early diagnosis and pre-onset therapies. In an interdisciplinary and longitudinal study,

\section{Badowska}

Department of Neurogenetics, Max Planck Institute for

Experimental Medicine, Hermann-Rein-Straße 3,

37075 Göttingen, Germany

\section{A. Schmitt $(\bowtie) \cdot$ P. Falkai}

Department of Psychiatry and Psychotherapy,

Ludwig-Maximilians-University Munich,

Nußbaumstr. 7, 80336 Munich, Germany

e-mail: Andrea.Schmitt@med.uni-muenchen.de
Toro et al. [2] found high total cholesterol in serum at the age of 60 to be associated with a higher risk of Alzheimer's disease and with mild cognitive impairment at the age of 75. The APOE\&4 allele, which is an important genetic risk factor for $\mathrm{AD}$, did not interact with serum cholesterol. In contrast to serum, cholesterol levels in cerebrospinal fluid did not correlate with the development of symptoms. Thus, total cholesterol in serum may be suitable as an early marker to predict the risk of disease in later life.

In a two-year follow-up fMRI study, Wolf et al. [3] recorded cortical deactivation changes in preclinical individuals carrying the Huntington's disease gene mutation (preHD) during verbal working memory tasks. No differences in region-specific deactivation were observed in preHD subjects compared to healthy controls, but functional connectivity was altered in several cortical regions, which remained stable over 2 years. In contrast, in the dorsal cingulate cortex, connectivity was increased over time in the preHD group and inversely correlated with the decline of motor functions. Thus, the investigation of tasknegative activity could shed light on time-sensitive brain functional processed associated in preHD carriers.

SLC6A2 encodes the norepinephrine transporter and may be associated with attention deficit hyperactivity disorder (ADHD) and autism spectrum disorders. Park et al. [4] investigated the influence of 10 single nucleotide polymorphisms (SNPs) in SLC6A2 on the phenotype of Korean autistic patients and their relatives. Although no significant associations were found, the authors assume that in a larger cohort of patients, a subtle effect of genotype on attention and repetitive behavior may be detected.

Schizophrenia and bipolar disorder display many genetical, pathophysiological and symptomatical similarities. In a series of neuropsychological tests, Melcher et al. [5] detected executive functions in general to be more 
severely affected in schizophrenia than in bipolar disorder which was confirmed by their findings that schizophrenia patients exhibited a more pronounced deficit in task performance, such as response, speed and accuracy. In addition, they displayed deficits in inhibitory control and attentional maintenance, which seems to be the hallmark of the disease distinguishing it from bipolar disorder. A study by Bachiller et al. [6] applied EEG-related spectral entropy and median frequency decreased during a P300 auditory odd-ball task to assess electrophysiological correlates with cognition. Schizophrenia patients displayed a lower decrease, which indicates greater irregularity of the EEG signal. Thus, spectral entropy can be relevant in studying cerebral processing in schizophrenia.

Psychosis and cognitive impairments accompanying Alzheimer's disease and subcortical ischemic vascular dementia (SIVD) may be associated with high levels of autoantibodies against the NR1 subunit of the NMDA receptor (NMDAR). Busse et al. [7] found an age-influenced increase of these antibodies in cerebrospinal fluid in healthy subjects, which again were not elevated in the cerebrospinal fluid of $\mathrm{AD}$ patients, but in serum-an effect which may accompany psychosis in these patients. It affords larger samples, to investigate whether these NMDAR serum autoantibodies potentially predispose patients with AD and SIVD to susceptibility for psychotic episodes, when the blood-brain barrier is affected.

Finally, deficits of insight in schizophrenia are associated with poor patient outcome. Psychological studies point out a link between insight and empathy in affected individuals. Bhagyavathi et al. [8] report that insight is independent of depression scores, cognitive insight and theory of mind. These findings support views of the individual human tendency to project own mental states on other people to predict their mental states. In other words, insight may be a requirement for empathy. However, the relationship between insight and empathy may depend on the severity of symptoms, proportion of schizoaffective to schizophrenia patients and gender bias.

\section{References}

1. Teipel SJ, Walter M, Likitjaroen Y, Schönknecht P, Gruber O (2014) Diffusion tensor imaging in Alzheimer's disease and affective disorders. Eur Arch Psychiatry Clin Neurosci. doi:10. 1007/s00406-014-0496-6

2. Toro P, Degen C, Pierer M, Gustafson D, Schröder J, Schönknecht P (2013) Cholesterol in mild cognitive impairment and Alzheimer's disease in a birth cohort over 14 years. Eur Arch Psychiatry Clin Neurosci. doi:10.1007/s00406-013-0468-2

3. Wolf RC, Sambataro F, Vasic N, Wolf ND, Thomann PA, Landwehrmeyer GB, Orth M (2013) Longitudinal task-negative network analyses in preclinical Huntington's disease. Eur Arch Psychiatry Clin Neurosci. doi:10.1007/s00406-013-0447-7

4. Park S, Park JE, Cho SC, Kim BN, Shin MS, Kim JW, Yoo HJ (2014). No association of the norepinephrine transporter gene (SLC6A2) and cognitive and behavioural phenotypes of patients with autism spectrum disorder. Eur Arch Psychiatry Clin Neurosci. doi:10.1007/s00406-013-0480-6

5. Melcher T, Wolter S, Falck S, Wild E, Wild F, Gruber E, Gruber O (2013) Common and disease-specific dysfunctions of brain systems underlying attentional and executive control in schizophrenia and bipolar disorder. Eur Arch Psychiatry Clin Neurosci. doi:10.1007/s00406-013-0445-9

6. Bachiller A, Díez A, Suazo V, Domínguez C, Ayuso M, Hornero R, Molina V (2014) Decreased spectral entropy modulation in patients with schizophrenia during a P300 task. Eur Arch Psychiatry Clin Neurosci. doi:10.1007/s00406-014-0488-6

7. Busse S, Busse M, Brix B, Probst C, Genz A, Bogerts B, Steiner J (2014) Seroprevalence of n-methyl-d-aspartate glutamate receptor (NMDA-R) autoantibodies in aging subjects without neuropsychiatric disorders and in dementia patients. Eur Arch Psychiatry Clin Neurosci. doi:10.1007/s00406-014-0493-9

8. Bhagyavathi HD, Mehta UM, Thirthalli J (2013) The relationship between empathy, emotion processing and clinical insight in remitted schizophrenia patients. Eur Arch Psychiatry Clin Neurosci. doi:10.1007/s00406-013-0484-2 